\title{
Extended crossing fibers of the mentalis muscle attaching to the contralateral mandible
}

\author{
Joe Iwanaga ${ }^{1,2,3}$, Mi-Sun Hur $^{4}$, Shogo Kikuta ${ }^{3}$, Soichiro Ibaragi ${ }^{5}$, R. Shane Tubbs ${ }^{1,2,6}$ \\ ${ }^{1}$ Department of Neurosurgery, Tulane Center for Clinical Neurosciences, Tulane University School of Medicine, New Orleans, LA, ${ }^{2}$ Department of \\ Neurology, Tulane Center for Clinical Neurosciences, Tulane University School of Medicine, New Orleans, LA, USA, ${ }^{3}$ Dental and Oral Medical Center, \\ Kurume University School of Medicine, Kurume, Fukuoka, Japan, ${ }^{4}$ Department of Anatomy, Catholic Kwandong University College of Medicine, \\ Gangneung, Korea, ${ }^{5}$ Department of Oral and Maxillofacial Surgery, Okayama University Graduate School of Medicine, Dentistry and Pharmaceutical \\ Sciences, Okayama, Japan, ${ }^{6}$ Department of Anatomical Sciences, St. George's University, St. George's, Grenada, West Indies
}

\begin{abstract}
Fibers of the facial muscles occasionally extend, cross the midline, and connect to surrounding structures on the contralateral side, perhaps enabling the mouth to make more delicate movements and generate more facial expressions. This case report describes a variant in which the extended fibers of the mentalis crossed the midline and indicates the relationship of these fibers to the surrounding structures. Some of the deepest fibers of the mentalis descended inferomedially and crossed transversely just below the chin prominence to attach to the periosteum of the mandible on the contralateral side. The variation presented in this study shed light on the interactions of the mentalis with the surrounding muscles.
\end{abstract}

Key words: Mentalis, Facial muscles, Anatomy

Received June 20, 2021; Accepted July 15, 2021

\section{Introduction}

Fibers of the facial muscles occasionally extend, cross the midline, and connect to surrounding structures on the contralateral side, perhaps enabling the mouth to make more delicate movements and generate more facial expressions [1]. In and around the chin area, the mentalis (MT), orbicularis oris, depressor anguli oris, depressor labii inferioris (DLI), and incisivus labii inferioris (ILI) muscles work together to protrude and retract the lower lip [1-4]. The MT, a muscle of the lower lip and chin, consists of upper and lower fibers and courses antero-posteriorly. The upper fibers are generally shorter than the lower fibers [2]. The MT fibers are also classified as medial and lateral. In both the upper and lower

\footnotetext{
Corresponding author:

Mi-Sun Hur (iD

Department of Anatomy, Catholic Kwandong University College of Medicine, Gangneung 25601, Korea

E-mail:mshur@cku.ac.kr
}

portions of the MT, the medial fibers descend and cross the midline to attach to the skin on the contralateral chin while the lateral fibers descend and attach to the skin on the ipsilateral chin. The ILI usually connects between the MT, the periosteum of the mandible, and the buccinator. The ILI fibers inserting into the MT do not cross the midline but terminate into the ipsilateral MT [2]. This case report describes a variant in which the extended fibers of the MT crossed the midline and indicates the relationship of these fibers to the surrounding structures.

\section{Case Report}

During routine dissection of a Korean male cadaver whose age at the time of death was 82 years, the MT and related muscles showed anatomical variations (Fig. 1). The lower face with muscle tissues was harvested en bloc with the periosteum. The tissues were then dissected posteriorly. When the course and connection of the MT fibers were examined, some of the deepest fibers were found to descend inferomedially and to cross transversely just below the chin 


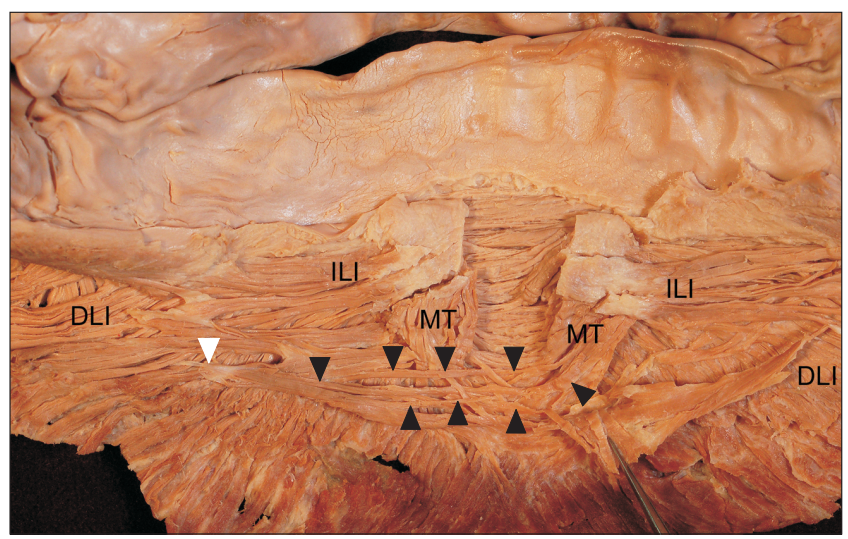

Fig. 1. Extended crossing fibers of the mentalis muscle (MT) attaching to the mandible of the contralateral side at the posterior aspect. Some of the deepest fibers (black arrowheads) of the MT descended inferomedially and crossed transversely just below the chin prominence to attach to the periosteum of the mandible on the contralateral side below the corner of the mouth by the thin aponeurosis (white arrowhead). After crossing the chin, these fibers were continuously arranged below the incisivus labii inferioris (ILI) on the contralateral side. Some of the deepest fibers of the MT were reflected inferiorly to reveal the extended crossing fibers of the muscle. DLI, depressor labii inferioris.

prominence to attach to the periosteum of the mandible on the contralateral side. These crossing fibers of the MT were intermingled with some of the deepest MT fibers on the contralateral side and were extended to the mandible below the corner of the mouth. After crossing the chin, they were continuously arranged below the ILI on the contralateral side. The other fibers of the MT showed the usual morphological patterns of this muscle. The medial fibers of the left and right MT crossed together, attaching to the chin skin of the contralateral side. Their lateral fibers descended inferomedially, intermingling with the DLI to attach to the skin of the ipsilateral side.

This study was conducted in accordance with the Declaration of Helsinki. The cadaver dissected had been legally donated to the Catholic Kwandong University College of Medicine. This study was approved by the Institutional Review Board of Catholic Kwandong University (IRB no. CKU21-01-0203). A surgical microscope (OPMI-FC; Carl Zeiss, Oberkochen, Germany) was used during detailed dissection on the deep surface of the removed facial muscles.

\section{Discussion}

Most human skeletal muscles generally originate from and insert into structures on the ipsilateral sides, but owing to their variability, many exceptions have been reported. Burley et al. (2020) [5] reported that sternal fibers of the pectoralis major crossed the midline. In Mori's study of Japanese cadavers, different musculatures were reported to have variants that crossed the midline, e.g., the anterior belly of the digastric muscle and the sternalis muscle [6]. Variant muscle fibers crossing the midline have therefore been well documented. A similar variant has also been reported in mimetic muscle [1].

The present study demonstrates a variation of the MT in which its extended fibers attached to the mandible on the contralateral side. These extended fibers passed just below the chin prominence, while the other crossing fibers reached the chin prominence to attach to its skin. These extended crossing fibers of the MT attached to the periosteum of the mandible of the contralateral side below the angle of the mouth instead of the skin of the chin. They might therefore contribute to making a longer sling that helps to support the chin prominence and submental fat.

The MT draws up the skin of the chin and thus indirectly causes the lower lip to protrude. It functions in articulation, in forcing bits of food from between the gums, and in the expression of various emotions such as pride and doubt [7]. Although it is a small muscle, it is located in the center below the lower lip and intermingles with several surrounding muscles such as the ILI, DLI, and platysma [2, 7], implying close anatomical and functional relationships with movements of the lower lip. Therefore, the variation presented in this study was considered in the light of the interactions of the MT with the surrounding muscles.

\section{ORCID}

Joe Iwanaga: https://orcid.org/0000-0002-8502-7952

Mi-Sun Hur: https://orcid.org/0000-0002-1482-1657

Shogo Kikuta: https://orcid.org/0000-0002-2236-2884

Soichiro Ibaragi: https://orcid.org/0000-0003-2897-1231

R. Shane Tubbs: https://orcid.org/0000-0003-1317-1047

\section{Author Contributions}

Conceptualization: JI, MSH. Data acquisition: MSH. Data analysis or interpretation: JI, MSH, SK, SI, RST. Drafting of the manuscript: JI, MSH, SK, SI, RST. Critical revision of the manuscript: JI, MSH, SK, SI, RST. Approval of the final version of the manuscript: all authors. 


\section{Conflicts of Interest}

No potential conflict of interest relevant to this article was reported.

\section{Acknowledgements}

The authors sincerely thank those who donated their bodies to science so that anatomical research could be performed. Results from such research can potentially increase mankind's overall knowledge that can then improve patient care. Therefore, these donors and their families deserve our highest gratitude [8].

This work was supported by the National Research Foundation of Korea (NRF) grant funded by the Korea government (MSIT) (No.2020R1C1C1003237).

\section{References}

1. Hur MS, Kim HJ, Lee KS. An anatomic study of the medial fibers of depressor anguli oris muscle passing deep to the depres- sor labii inferioris muscle. J Craniofac Surg 2014;25:614-6.

2. Hur MS, Kim HJ, Choi BY, Hu KS, Kim HJ, Lee KS. Morphology of the mentalis muscle and its relationship with the orbicularis oris and incisivus labii inferioris muscles. J Craniofac Surg 2013;24:602-4.

3. Iwanaga J, He P, Watanabe K, Kamura Y, Oskouian RJ, Tubbs RS. Intraoral observation of the mentalis and incisivus labii inferioris muscles. J Craniofac Surg 2017;28:2159-61.

4. Standring S. Gray's anatomy: the anatomical basis of clinical practice. 42nd ed. London: Elsevier; 2020.

5. Burley H, Georgiev GP, Iwanaga J, Dumont AS, Tubbs RS. An unusual finding of the pectoralis major muscle: decussation of sternal fibers across the midline. Anat Cell Biol 2020;53:505-8.

6. Mori M. Statistics on the musculature of the Japanese. Okajimas Folia Anat Jpn 1964;40:195-300.

7. Morris H. Human anatomy: a complete systematic treatise. 10th ed. Philadelphia: Blakiston; 1947.

8. Iwanaga J, Singh V, Ohtsuka A, Hwang Y, Kim HJ, Moryś J, Ravi KS, Ribatti D, Trainor PA, Sañudo JR, Apaydin N, Şengül G, Albertine KH, Walocha JA, Loukas M, Duparc F, Paulsen F, Del Sol M, Adds P, Hegazy A, Tubbs RS. Acknowledging the use of human cadaveric tissues in research papers: recommendations from anatomical journal editors. Clin Anat 2021;34:24. 